\title{
A GENERALIZATION OF A THEOREM OF TOTTEN
}

\author{
R. C. MULLIN and S. A. VANSTONE
}

(Received 22 December 1975; revised 10 February 1976)

\begin{abstract}
An $(r, 1)$ system is pair $(V, F)$ where $V$ is a $v$-set and $F$ is a family of non-null subsets of $V(b$ in number) which satisfy the following.

(i) Every pair of distinct members of $V$ occur in precisely one member of $F$;

(ii) Every member of $V$ occurs in precisely $r$ members of $F$.

A pseudo parallel complement $\operatorname{PPC}(n, \alpha)$ is an $(n+1,1)$ system with $v=n^{2}-\alpha n$ and $b \leqq$ $n^{2}+n-\alpha$ in which there are at least $n-\alpha$ blocks of size $n$. A pseudo intersecting complement $P I C(n, \alpha)$ is an $(n+1,1)$ system with $v=n^{2}-\alpha n+\alpha-1$ and $b \leqq n^{2}+n-\alpha$ in which there are at least $n-\alpha+1$ blocks of size $n-1$. It is shown that for $\alpha \geqq 4$, every $\operatorname{PIC}(n, \alpha)$ can be embedded in a $\operatorname{PPC}(n, \alpha-1)$ and that for $n>\left(a^{4}+2 \alpha^{3}+2 \alpha^{2}+3 \alpha\right) / 2$ every $P P C(n, \alpha)$ can be embedded in a projective plane of order $n$. The latter generalizes a result of Totten (who proves the resuli for $\alpha=1$ ).
\end{abstract}

\section{Introduction}

Totten (1976) has proved that for $n \geqq 5$, the complement of a line in an affine plane of order $n$ can be embedded in a finite projective plane of order $n$. To be more precise, we require the following definitions.

A finite linear space is a finite set of $p$ objects, called points together with a (finite) set of $q$ subsets of points, called lines, such that every pair of distinct points is included in precisely one line and every line contains at least two points. The degree of a line (respectively point) is the number of points which it contains (respectively is contained in). For definition of projective and affine planes see Blake and Mullin (1975). In this terminology, Totten proves that if $n$ is a positive integer $>4$, then any finite linear space having $p=n^{2}-n$ points, each of degree $n+1$ and $q \leqq n^{2}+n-1$ lines is embeddable in a finite projective plane of order $n$. Clearly such a configuration can be obtained by deleting a line in an affine plane of order $n$. Let us note that by deleting the points on $\alpha$ lines from a parallel class in an affine plane of order $n>\alpha+2$, one obtains a finite linear space with at most $n^{2}+n-\alpha$ lines, at least $n-\alpha$ of 
which have degree $n$ and in which the number of points is $n^{2}-n \alpha$. Moreover each of the points has degree $n+1$. We show that for $n>$ $\left(\alpha^{4}+2 \alpha^{3}+2 \alpha^{2}+3 \alpha\right) / 2$, such a configuration can be embedded in an affine plane of order $n$. The proof follows directly from a theorem of Bruck (1963). This result is extended to the case of a finite linear space with $p=$ $n^{2}-\alpha n+\alpha-1$ points, each of degree $n+1$, and $q \leqq n^{2}+n-\alpha$ lines, at least $n-\alpha+1$ of which have degree $n-1$; that is, the complement of a pencil of $\alpha \geqq 4$ lines through a common point in an affine plane of order $n$.

\section{Design terminology}

Since the various results employed in the proof of the main theorem here are stated in design theoretic language, we reformulate the problem in those terms.

An $(r, \lambda)$ system is a pair $(V, F)$ where $V$ is a finite set of $v$ elements called varieties, and $F$ is a family of non-null subsets of $V$ which satisfies the following axioms:

(i) every variety of $V$ occurs in precisely $r$ members of $F$,

(ii) every pair of distinct varieties occurs in precisely $\lambda$ members of $F$.

The symbol $b$ is used to denote the number of subsets in $F$, and the word cardinality is replaced by the word size. Clearly every finite linear space in which every point is of degree $r$ is an $(r, 1)$ system. Conversely, any $(r, 1)$ system with no blocks of size 1 is a finite linear space, as is any $(r, 1)$ system from which the blocks of size 1 have been removed.

\section{The complement of a parallel pencil}

We refer to any $(n+1,1)$ system with $v=n^{2}-n \alpha$ and $b \leqq n^{2}+n-\alpha$ which contains at least $n-\alpha+1$ lines of degree $n$ as a pseudo parallel complement $\operatorname{PPC}(n, \alpha)$.

We prove the main result of this section after a series of lemmas.

Lemma 3.1. Let $D$ be an $(n+1,1)$ system with $v=n^{2}-n \alpha$ and $b \leqq$ $n^{2}+n-\alpha$. If $n>\alpha^{2}+\alpha-1$, then $b=n^{2}+n-\alpha$.

Proof. It is shown in Mullin and Vanstone $(1976)$ that in any $(r, 1)$ system the number of blocks $b$ satisfies

$$
b \geqq r^{2} v /(r+v-1) .
$$

In the present situation this reduces to

$$
b \geqq n^{2}+n-\alpha-\alpha^{2} /(n-\alpha+1) .
$$


Lемма 3.2. Let $\alpha$ be any non-negative integer and $n$ any integer satisfying $n \geqq \alpha+1$. Let $D$ be an $(n+1,1)$ system with $v=n^{2}-n \alpha$ varieties and $b=n^{2}+n-\alpha$ blocks, at least $n-\alpha$ of which contain at least $n$ varieties. Then $D$ contains precisely $n-\alpha$ blocks of size $n$ and the remaining $n^{2}$ blocks are all of size $n-\alpha$.

Proof. Since we have assumed that $\alpha$ is non-negative, the total number of blocks is less than $n^{2}+n+1$. Since a block of size $n+1$ or greater in $D$ implies that there are at least $n^{2}+n+1$ blocks in $D$, clearly the maximum block size is $n$. Now let the blocks be denoted by $B_{1}, B_{2}, \cdots, B_{b}$ where blocks $B_{n^{2}+1}, B_{n^{2}+2}, \cdots, B_{b}$ are of size $n$. Let $k_{i}=\left|B_{i}\right|, i=1,2, \cdots, n^{2}$. Then elementary counting arguments establish the following equations.

$$
\sum_{i=1}^{n^{2}} k_{i}=n^{3}-n^{2} \alpha
$$

$$
\sum_{i=1}^{n^{2}} k_{i}^{2}=n^{2}(n-\alpha)^{2} \text {. }
$$

From this we obtain

$$
\sum_{i=1}^{n^{2}}\left(k_{i}-n+\alpha\right)^{2}=0
$$

which shows that $k_{i}=n-\alpha, i=1,2, \cdots, n^{2}$.

We say that an $(n+1,1)$ system $D_{1}$ can be embedded in an $(n+1,1)$ system $D_{2}$ if there exists a subset $W$ of the variety set of $D_{2}$ such that the restriction of $D_{2}$ to $W$ is (isomorphic to) $D_{1}$.

THEOREM 3.3. (Main Theorem). Let $\alpha$ be any non-negative integer and $n$ any integer satisfying $n>\alpha+1$. Let $D$ by any $\operatorname{PPC}(n, \alpha)$. If $n>$ $\left(\alpha^{4}+2 \alpha^{3}+2 \alpha^{2}+3 \alpha\right) / 2$, then $D$ can be embedded in an affine plane of order $n$.

Proof. Let $x$ be any variety of $D$. Let $r_{i}$ denote the number of blocks of size $i$ which contain $x$. Then by Lemma 3.2, $r_{i}=0$ unless $i=n-\alpha$ or $i=n$. Also we have the following:

(i) $r_{n-\alpha}+r_{n}=n+1$,

(ii) $(n-\alpha-1) r_{n-\alpha}+(n-1) r_{n}=n^{2}-n \alpha-1$.

From these we obtain $r_{n-\alpha}=n$ and $r_{n}=1$. Hence every variety occurs in precisely one block of size $n$. If $B_{1}, B_{2}, \cdots, B_{n-\alpha}$ denote the blocks of size $n$, then the remaining blocks form a transversal system (see, for example, Blake and Mullin (1975)) on the sets $B_{1}, B_{2}, \cdots, B_{n-\alpha}$. However, it is known (ibid.) 
that such a transversal system is equivalent to an orthogonal array $O A(n, n-$ $\alpha)$. However Bruck (1963) has proved that if $n>\left(\alpha^{4}+2 \alpha^{3}+2 \alpha^{2}+3 \alpha\right) / 2$ then any $O A(n, n-\alpha)$ can be embedded in an $O A(n, n+1)$. In terms of transversal systems this implies that the system defined on sets $B_{1}, B_{2}, \cdots, B_{n-\alpha}$ as above can be embedded in a transversal system on sets $B_{1}, B_{2}, \cdots, B_{n+1}$, which trivially implies the existence of a projective plane containing these sets and their associated transversal system. Moreover the embedding of the system in a transversal system as above shows that the system can be embedded in a transversal system defined on the sets $B_{1}, B_{2}, \cdots, B_{n}$, which together with these sets constitutes an affine plane of order $n$. If $\alpha=0$, then the first observation is merely that any affine plane of order $n$ can be embedded in a projective plane of order $n$. For $\alpha \geqq 0$, the second observation is that the system can be embedded in an affine plane of order $n$.

Corollary. Let $n$ and $\alpha$ be as above and $D$ any $\operatorname{PPC}(n, \alpha)$. If $n>$ $\left(\alpha^{4}+2 \alpha^{3}+2 \alpha^{2}+3 \alpha\right) / 2$, then $D$ can be embedded in an affine plane of order $n$.

Proof. This follows from Lemma 3.1 and the above theorem once one notes that $\alpha^{4}+2 \alpha^{3}+2 \alpha^{2}+3 \alpha>\alpha^{2}+\alpha-1$ for $\alpha \geqq 0$.

In terms of finite linear spaces, the preceding corollary becomes

THEOREM 3.3A. Let $\alpha$ be any positive integer and $n$ be any integer satisfying $n \geqq \alpha+2$. Let $L$ be a finite linear space with $p=n^{2}-\alpha n$ points, each of degree $n+1$, and $q \leqq n^{2}+n-\alpha$ lines, at least $n-\alpha$ of which have degree at least $n$. If $n>\left(\alpha^{4}+2 \alpha^{3}+2 \alpha^{2}+3 \alpha\right) / 2$, then $L$ can be embedded in an affine plane of order $n$.

The above becomes Totten's theorem on putting $\alpha=1$ and noting that in any linear space with $n^{2}-n$ points of degree $n+1$ and no more than $n^{2}+n-1$ lines, there are at least $n-1$ lines of degree $n$.

\section{The complement of an intersecting pencil}

In this section we consider configurations which arise in the following way. Let $L$ be be a finite affine plane and $M$ a set of $\alpha<n-1$ lines which intersect in a common point. Then the restriction of $L$ to the points not occurring in $M$ is an $(n+1,1)$ design with $n^{2}-\alpha n+\alpha-1$ varieties and $b \leqq n^{2}+n-\alpha$ blocks, at least $n-\alpha+1$ of which are of size $n-1$.

We refer to any $(n+1,1)$ design with $v=n^{2}-\alpha n+\alpha-1$ varieties and $b \leqq n^{2}+n-\alpha$ blocks, at least $n-\alpha+1$ of which are of size $n-1$ as 
a pseudo intersecting complement and denote such a configuration by $\operatorname{PIC}(n, \alpha)$. We shall show that for $\alpha \geqq 4$, every $\operatorname{PIC}(n, \alpha)$ can be embedded in a $\operatorname{PPC}(n, \alpha-1)$.

Lemma 4.1. Let $\alpha$ be any integer $\geqq 3$ and $D$ be any $\operatorname{PIC}(n, \alpha)$. Then $D$ can be embedded in a PPC $(n, \alpha-1)$ if and only if any pair of distinct blocks of size $n-1$ in $D$ are disjoint.

Proof. The necessity is easily established. Conversely if any pair of distinct blocks are disjoint, then any set on $n-\alpha+1$ lines of size $n-1$ contain all varieties precisely once. Hence there are precisely $n-\alpha+1$ such. Since each variety occurs once in these blocks, we can adjoin a new variety, say $x$, to each of these blocks, and adjoin $\alpha$ blocks consisting of $x$ only, to $D$ to obtain an $(n+1,1)$ system $D_{1}$ which contains $n-\alpha+1$ blocks of size $n$ and has $b_{1} \leqq n^{2}+n+1$ blocks. Vanstone (1973) has shown that any $(n+1,1)$ design on $v$ varieties which contains a block of size $n$ and has $n^{2}+n+1$ or fewer blocks can be extended to an $(n+1,1)$ design on $v+1$ varieties which contains $n^{2}+n+1$ or fewer blocks and in making this extension the adjoined variety can be made to occur in any specified block of size $n$.

Applying this result $n-\alpha+1$ times one can obtain an $(n+1,1)$ design $D_{2}$ on $n^{2}-(\alpha-1) n+1$ varieties in which there are $n-\alpha+1$ blocks of size $n+1$ each containing $x$ and one of the newly adjoined varieties. Hence none of the added varieties can be adjoined to a block consisting only of $x$ since each occurs with $x$ in a block of size $n+1$. We construct a design $D_{3}$ from $D_{2}$ as follows. Let $V_{2}$ denote the variety set of $D_{2}$. Then $D_{3}$ is the restriction of $D_{2}$ to $D_{2}$ to $V_{2}-\{x\}$. Clearly $D_{3}$ is an $(n+1,1)$ system on $n^{2}-(\alpha-1) n$ varieties which contains $n-(\alpha-1)$ blocks of size $n$. Moreover the removal of $\alpha$ singleton blocks from $D_{2}$ shows that $b \leqq$ $n^{2}+n-(\alpha-1)$. In other words, $D_{3}$ is a $\operatorname{PPC}(n, \alpha-1)$.

Lemma 4.2. Let $D$ be an $(n+1,1)$ system with $b \leqq n^{2}+n-\alpha$ blocks. If $\alpha \geqq 4$, then any pair of distinct blocks of size $n-1$ are disjoint.

Proof. Assume that some such design contains blocks $B_{1}$ and $B_{2}$ which intersect in some variety, and that $B_{1} \neq B_{2}$. Then the varieties of $B_{1} \cup B_{2}$ occur in $n^{2}+n-3$ blocks, contradicting $\alpha \geqq 4$.

Theorem 4.3. Let $D$ be any $\operatorname{PIC}(n, \alpha)$ with $\alpha \geqq 4$. Then $D$ can be embedded in a PPC $(n, \alpha-1)$. Further, if $n>\left(\alpha^{4}-2 \alpha^{3}+2 \alpha^{2}+a-2\right) / 2$, then $D$ can be embedded in an affine plane of order $n$. 
Proof. The embedding of $D$ in a $P P C(n, \alpha-1)$ follows from lemmata 4.1 and 4.2. The embedding of $D$ in an affine plane for $n>$ $\left(\alpha^{4}-2 \alpha^{3}+2 \alpha^{2}+\alpha-2\right) / 2$ follows from the embedding of a PPC $(n, \alpha-1)$ in an affine plane in accordance with theorem 3.3 .

We show that not every $\operatorname{PIC}(n, 3)$ can be embedded in a $P P C(n, 2)$. Indeed, consider the $\operatorname{PIC}(4,3)$ listed below.

$\begin{array}{rrrrr}123 & 34 & 36 & 1 & 5 \\ 145 & 35 & 46 & 2 & \\ 24 & 16 & 56 & 3 & \\ 25 & 26 & 1 & 4 & \end{array}$

It contains two blocks of size 3 which intersect. Hence it cannot be embedded in a $P P C(4,2)$.

In terms of finite linear spaces, Theorem 4.3 becomes the following. Let $L$ be a finite linear space with $p=n^{2}-n+\alpha-1$ points and $q \leqq n^{2}+n-\alpha$ lines, where $\alpha$ is an integer exceeding 3 and $n \geqq\left(\alpha^{4}-2 \alpha^{3}+2 \alpha^{2}+\alpha-2\right) / 2$. If each point of $L$ has degree $n+1$ and there are at least $n+\alpha-1$ lines of degree $n-1$, then $L$ can be embedded in a projective plane of order $n$.

An example of a finite linear space which is not embeddable in the corresponding affine plane is the following $\operatorname{PIC}(5,3)$ listed below.

$\begin{array}{ccc}1234 & 49 \mathrm{E} & 47 \\ 1567 & 5 \mathrm{VE} & 18 \\ 2589 & 68 \mathrm{E} & 19 \\ 36 \mathrm{~T} & 78 \mathrm{~V} & 1 \mathrm{~V} \\ 37 \mathrm{E} & 79 \mathrm{~T} & 2 \mathrm{E} \\ 46 \mathrm{~V} & 2 \mathrm{TV} & 38 \\ 1 \mathrm{TE} & 27 & 5 \mathrm{~T} \\ 39 \mathrm{~V} & 35 & 69 \\ 48 \mathrm{~T} & 45 & 26\end{array}$

A PIC $(4,2)$ which is a finite linear space and which is not embeddable in an affine plane of order 4 can also be readily produced. 


\section{References}

I. F. Blake and R. C. Mullin (1975), The Mathematical Theory of Coding, (Academic Press, New York).

R. H. Bruck (1963), 'Finite nets II, uniqueness and embedding', Pacific J. Math. 13, 421-457.

R. C. Mullin and S. A. Vanstone (1976), 'On regular pairwise balanced designs of order 6 and index 1', Utilitas Math.

S. A. Vanstone (1973), 'The extendibility of $(r, 1)$ designs', Proc. Third Southeastern Conference on Numerical Math., Utilitas Math. Inc., Winnipeg, 409-418.

J. Totten (1976), 'Embedding the complement of two lines in a finite projective plane', $J$. Austral. Math. Soc. 22, 27-34.

Department of Combinatorics, University of Waterloo, Ontario, Canada. 